\title{
Immunological Reactions in Atopic Dermatitis and Possible Improvement of Probiotics
}

\author{
Sur Genel ${ }^{1,2 *}$, Sur M Lucia ${ }^{1}$, Sur Daniel ${ }^{1}$, Coroian Aurelia ${ }^{3}$ and Floca Emanuela ${ }^{1}$ \\ ${ }^{1}$ University of Medicine and Pharmacy, IuliuHatieganu, Cluj-Napoca, Romania \\ ${ }^{2}$ Emergency Clinical Hospital for Children, Cluj-Napoca, Romania \\ ${ }^{3}$ Faculty of Animal Science and Biotechnology, University of Agricultural Sciences and Veterinary Medicine Cluj-Napoca, Romania
}

\begin{abstract}
Allergic diseases are common hyper-immune disorders. Atopic dermatitis (AD) has a higher prevalence in industrialized countries. It has been suggested that association between genetic factors, environmental factors and gut microbiota would be major triggering factors for the development of atopic dermatitis. Hygiene hypothesis by a lack of early childhood exposure to diverse microorganism increases susceptibility to atopic dermatitis and other allergic diseases. Recent studies have demonstrated the complex interrelationship between skin barrier, genetic, environmental, pharmacologic, psychological and immunologic factors that contribute to the development and severity of $A D$. The current review will examine the immunological mechanism that contributes to $A D$ as well as immunologic triggers involved in the pathogenesis.
\end{abstract}

Keywords: Atopic dermatitis; Probiotics; Immunology

\section{Introduction}

Atopic dermatitis (AD) is an inflammatory skin disease with early onset. The prevalence of AD is approximately $20 \%$. Dermatitis and eczema are often used synonymously although the term eczema is sometimes reserved for acute manifestations [1].

\section{Pathophysiology}

Two hypotheses have been proposed to explain the inflammatory lesions in $\mathrm{AD}$ : immunological hypothesis and the skin barrier hypothesis. The theory of immunological imbalance argues that $\mathrm{AD}$ result from an imbalance of $\mathrm{T}$ helper cell types 1,2,17,22 and also regulatory $\mathrm{T}$ cells $[2,3]$. In $\mathrm{AD}$ the $\mathrm{Th} 2$ differentiation of naive CD4 $\mathrm{T}$ cells predominates, thus causing an increased production of interleukins, primarily IL-4, IL-5, IL-13 which then leads to an increased level of $\operatorname{IgE}$ and Th1 differentiation is inhibited. The theory of skin barrier sustains that mutations in the filaggrin gene increase the risk of developing AD. The filaggrin gene encodes structural proteins in the stratum corneum and stratum granulosum that helps bind the keratinocytes together. With gene defects less filaggrin is produced leading to skin barrier dysfunction $[2,4]$.

\section{The role of cytokines in AD skin lesion}

Th2 and Th1 cytokines contribute to the pathogenesis of skin inflammation in AD. Acute T-cell infiltration in AD is associated with a predominance of IL4-IL13 expression. Chronic inflammation is associated with increased IL-5, GM CSF, IL-12 and IFN $\gamma$ accompanied by the infiltration of eosinophils and macrophages. Il12 in chronic AD skin lesions is very important because that cytokine play a key role in Th1 cell development and its expression in eosinophil or macrophages is thought to initiate the switch to Th1 cell development in chronic AD. The activity of cytokines depends on the expression of their receptor. In acute $\mathrm{AD}$ lesions it was found a significant higher number of cells expression of IL-4R-a mRNA compared with chronic AD which contained significantly more cells expressing the IL-5R- $\alpha$ and GM-CSFR- $\alpha$. This biphasic pattern of T-cell activation has also been demonstrated in studies of allergen patch testing $[4,5]$.

\section{Chemoattractant factors}

Studies have demonstrated that IL16, a chemoattractant for CD4 $\mathrm{T}$ cells, is more highly expressed in acute than in chronic AD skin lesions. Another chemoattractant factors like such as $\mathrm{C}$-C chemokines, RANTES and eotoxin have also been found to be increased in AD and contribute to the chemotaxis of eosinophils and Th2 lymphocytes into the skin. Chronic AD is linked to the prolonged survival of eosinophil and monocyte-macrophage in atopic skin $[1,3]$.

\section{From acute to chronic atopic dermatitis}

The rise in IL-5 expression during the transition from acute to chronic $\mathrm{AD}$ plays a role in the prolongation of eosinophil survival in chronic AD. For survival and function of monocytes GM-CSF play an important role. IL-4 also supports the maturation of monocytes into dendritic cells. Epidermal Keratinocyes when stimulate simultaneously with IFN $\gamma$ and TNFa were found to produce increased level of RANTES which enhanced the chemotaxis of eosinophils. This is one mechanism by which the rise in IFN $\gamma$ during chronic $\mathrm{AD}$ enhances the chronicity and severity of AD. Mechanical trauma can induce the release of TNFa and other pro-inflammatory cytokines from epidermal keratinocytes. However chronic AD is frequently associated with colonization by super antigens producing Staphylococus aureus $[3,6,7]$.

\section{Role of probiotics in atopic dermatitis}

Atopic manifestations have been described throughout history since ancient China. Roman Emperor Octavianus Augustus was

*Corresponding author: Sur Genel, University of Medicine and Pharmacy, luliuHatieganu, 8 Babeş Street, 400012 Cluj-Napoca, Romania, Tel: +40 264597 256; E-mail: surgenel@yahoo.com

Received August 31, 2015; Accepted September 25, 2015; Published September 28,2015

Citation: Genel S, Lucia SM, Daniel S, Aurelia C, Emanuela F (2015) Immunological Reactions in Atopic Dermatitis and Possible Improvement of Probiotics. Pharm Anal Acta 6: 420. doi:10.4172/21532435.1000420

Copyright: ( $) 2015$ Genel S, et al. This is an open-access article distributed unde the terms of the Creative Commons Attribution License, which permits unrestricted use, distribution, and reproduction in any medium, provided the original author and source are credited. 
Citation: Genel S, Lucia SM, Daniel S, Aurelia C, Emanuela F (2015) Immunological Reactions in Atopic Dermatitis and Possible Improvement of Probiotics. Pharm Anal Acta 6: 420. doi:10.4172/21532435.1000420

Page 2 of 2

the first person to which was described atopic manifestations. The production mechanism of atopy remained a mystery to the nowadays.

Hipocrates has been quoted as saying "death sits in the bowels" and "bad digestion is the root of all evil" showing that the importance of the intestines in human health has been long recognized.

The gut microbiota may also be involved in the etiology of atopic dermatitis. The atopic diseases are chronic inflammatory disorders caused by aberrant $\mathrm{T}$ helper 2 (Th2) immune responses against common innocuous environmental antigens in susceptible individuals. The importance of a delicate balance between allergen - specific T-reg cells and allergen-specific Th2 cells in healthy and allergic immune responses to common environmental allergen was demonstrated in study of Atkis et al. [8,9].

Probiotics could stimulate the immune system by modulating the composition or activity of the intestinal microbiota. Some probiotics generate IL $10, \operatorname{Tr} 1, \mathrm{CD} 4$ other producing IL1 $\beta$, IL12, TNFa. Lactobacillus reuteri induces the production of IL12 and YNFa while Lactobacillus casei inhibits the production of pro-inflammatory cytokines by producing anti inflammatory cytokines IL10. It is crucial to use specific probiotics or their mixture to target specific immune disorders. IRT5 probiotics, a mixture of 5 probiotics, could suppress diverse immune disorders through the generation of CD4 and administration of IRT5 probiotics suppressed ongoing experimental $\mathrm{AD}$. It is very important to identify potent probiotics that could induce the production of immune-modulatory cytokines such as IL10 and TGF $\beta$ or enhancing Th 1 response to improve the symptoms of allergic disease $[8,10,11]$. Perturbation in the intestinal microbiota may disrupt mechanism involved in the development of immunologic tolerance. The microbiota hypothesis in atopic diseases is promising. The 17 observational studies conducted indicate an association between the gut microbiota composition and atopic sensitization or symptoms. Probiotics confer a health benefit on the host. Recent studies showed that probiotics can also exert beneficial effects by directly modulating immune system. The treatment with certain probiotics may prevent and improve symptoms of experimental atopic dermatitis, inflammatory bowel disease and asthma by down regulating inflammatory cytokines inducing immune regulatory mechanism $[9,11]$.

The Bifidobacteria from atopic infants induces high levels of proinflammatory cytokine in vitro while the Bifidobacteria from non atopic infants induces more secretion of anti inflammatory cytokines.
Environmental factors in addiction to hygiene hypothesis and intestinal microflora contribute to allergic disease by substantially affecting the mucosal immunity [11].

Many randomized controlled trials have investigated the effect of probiotics on the prevention of AD. Probiotics were given to infants with a high risk of developing allergy, starting immediately after birth. Mothers also received probiotics during the last week of pregnancy. The trial has reported a $50 \%$ reduction in the incidence of $A D$ in the probiotics group compared to placebo group at the age of 2 years [12].

\section{References}

1. Mark Boguniewicz, Donald YM Leung (2011) Atopic dermatitis: A disease of altered skin barrier and immune dysregulation. Immunological Reviews 242: 233-246.

2. Karagiannidou A, Botskariova S, Farmaki E, Imvrios G, Mavroudi A (2014) Atopic Dermatitis: Insights on Pathogenesis, Evaluation and Managemen. J Allergy Ther 5.

3. Kenji Kabashima (2013) New concept of the pathogenesis of atopic dermatitis: Interplay among the barrier, allergy, and pruritus as a trinity. J Dermatol Sci 70: 3-11.

4. Eric B Brandt, Sivaprasad U (2011) Th2 Cytokines and Atopic Dermatitis. J Clin Cell Immunol 2.

5. Kuo IH, Yoshida T, De Benedetto A, Beck LA (2013) The cutaneous innate immune response in patients with atopic dermatitis. J Allergy Clin Immunol 131 266-278.

6. Howell MD, Kim BE, Gao P, Grant AV, Boguniewicz M, et al. (2007) Cytokine modulation of atopic dermatitis filaggrin skin expression. J Allergy Clin Immunol 124: $150-155$

7. Wolf $\mathrm{R}$, Wolf $\mathrm{D}$ (2012) Abnormal epidermal barrier in the pathogenesis of atopic dermatitis. Clin Dermatol 30: 329-334.

8. Novak N, Simon D (2011) Atopic dermatitis - from new pathophysiologic insights to individualized therapy. Allergy 66: 830-839.

9. Ring J, Alomar A, Bieber T, Deleuran M, Fink-Wagner A, et al. (2012) Guidelines for treatment of atopic eczema (atopic dermatitis) Part II. J Eur Acad Dermatol Venereol 26: 1176-1193.

10. Sur Genel, Sur M Lucia, Sur Daniel, Floca Emanuela (2015) Can the Probiotics Change the Spectrum of Atopic Dermatitis? Pharmaceutica Analytica Acta 6.

11. Yesilova Y, calka O, Akdeniz N, Berktas M (2012) Effect of Probiotics on the Treatment of Children with Atopic Dermatitis. Annals of Dermatology 24: 189193

12. Pelucchi C, Chatenoud L, Turati F, Galeone C, Moja L, et al. (2012) Probiotics Supplementation During Pregnancy or Infancy for the Prevention of Atopic Dermatitis: A Meta-analysis. Epidemiology 23: 402-414. 